\title{
The Surrogate Safety Appraisal of the Unconventional Elliptical and Turbo Roundabouts
}

\author{
Giovanni Tesoriere, ${ }^{1}$ Tiziana Campisi ${ }^{D},{ }^{1}$ Antonino Canale, ${ }^{1}$ and Tedi Zgrablic ${ }^{2}$ \\ ${ }^{1}$ Faculty of Engineering and Architecture, University of Enna Kore, Italy \\ ${ }^{2}$ Faculty of Engineering and Architecture, University of Maribor, Slovenia \\ Correspondence should be addressed to Tiziana Campisi; tiziana.campisi@unikore.it
}

Received 2 May 2018; Revised 26 August 2018; Accepted 5 September 2018; Published 9 October 2018

Guest Editor: Daiva Zilioniene

Copyright (c) 2018 Giovanni Tesoriere et al. This is an open access article distributed under the Creative Commons Attribution License, which permits unrestricted use, distribution, and reproduction in any medium, provided the original work is properly cited.

\begin{abstract}
Double-lane roundabouts have been created in many European countries over the past few centuries and are now characterized by an unsafe geometric development and by a low sustainability capacity or level. In this regard, new double-lane geometries have been implemented to overcome to these critical points. This article shows a comparison of two nonconventional doublelane roundabout schemes defined as elliptical and turbo. Considering this research on the unsafe and congested conditions for each road schemes at grade, the microsimulation approach allows comparing schemes of intersections not yet realized in order to be able to evaluate the critical issues. A symmetric traffic distribution and an identical vehicle mix for both design solutions are considered. The research was conducted considering two different double-lane roundabout-turbo roundabout and the elliptical roundabout. By comparing their geometry and technical elements, this article assumes that turbo roundabout due to its physical separating traffic lanes in the central circulatory carriageway will enable potentially better traffic safety conditions. This article has the following main goal: a comparison of traffic safety using VISSIM microsimulator and SSAM tools. The results can provide to show safety level on investigated scenario considering level of service (LOS) and also the possibility of obtaining time to collision (TTC) and postencroachment time (PET) through the use of surrogate parameters obtained by SSAM tool. In fact, the surrogate safety parameters allows evaluating the possible collision scenarios between them, according to the trajectories of the single vehicles. This assessment is useful in order to be able to evaluate by the local authorities which of the examined schemes can provide greater negativity in the construction and operation phase. Therefore this comparative analysis allows reducing, in the preliminary phase, possible security impacts and also economic ones for the community.
\end{abstract}

\section{Introduction}

Today, the different road intersections are increasingly developing. In general, the road intersections in roundabout allow a substantial reduction of the points of conflict between vehicles and vehicles with pedestrians compared to the traditional intersections with precedence or with traffic lights. Proper planning of the roundabout is necessary to reduce the percentage of deaths and injuries on the roads of each countries.

The research was conducted considering two different double-lane roundabouts titled elliptical and turbo. The following paragraphs show a geometric description of the intersections and a detailed analysis of the steps that led to the comparison of the two geometries in safety terms through the use of the VISSIM microsimulator [1] and the dedicated SSAM tool [2].

There was the first phase of selection of the geometries to be analyzed, the traffic scenarios with relative distribution of the vehicle typologies and after the calibration of the model of the microsimulator in order to make the most reliable results. Finally, they were processed in terms of surrogate safety through a dedicated tool.

The comparison of the results shows how to vary the flows and geometries of unconventional satin road intersection you can have more or fewer distributions of certain types of collisions between vehicles along the geometry. In fact, the evaluation and also the comparison of supposed collision 
are investigated considering surrogate parameters. Surrogate parameters represent a summary assessment in terms of safety, by placing a double-lane rotary comparison on the central circulatory carriageway.

The hypotheses on which this research is based are related to the definition of the surrogate safety of nonconventional roundabout geometries. Specifically, attention was focused on the comparison of two widespread schemes in Europe, in order to be able to investigate in advance which of these, with the same outflow conditions, be better in terms of service level and therefore road safety.

\section{Double-Lane Roundabout Geometry}

The geometries chosen for the comparison are of a satin type and are referred to as turbo and elliptical roundabout, respectively. Both have in the case examined a double lane in the central circulatory carriageway. At the end of the $90 \mathrm{~s}$, in the Netherlands it is following the design and construction of the turbo-type roundabouts. Scheme of stories has evolved over the years by presenting the preselection of the manoeuvres along the arms and therefore they are vehicles of not manoeuvring abrupt or wrong.

The limitation of the manoeuvres was made possible not only by the preselection along the lanes of the arms but also by the presence of curbs or signs of separation between the manoeuvres along the main ring. From here there has been an increase in safety with a reduction in the number of deaths and injuries along these intersecting geometries. Fortuijn was the first to study and design the turbo-type intersections at grade [3].

Unlike the turbo roundabout, the elliptic schemes are not defined by separated lanes of the circulatory ring. Sometimes there are not even the preselection lanes along one or more arms of the intersection.

Several studies have been conducted in recent years on the assessment of the potential of unconventional roundabout in terms of safety and environmental impact, evaluating both the single and double lane on the central ring in different traffic flow conditions like those described by $[5,6]$.

The evolution of turbo-type rotary geometries has in recent years been carried out in parallel with the development of public and private transport systems in terms of both ecomobility and traffic decongestion systems with the reduction of the automatic system in favour of collective passengers transport [7].

The elliptical roundabout is a form of a classical doublelane roundabouts with the emphasis that it is not a proper circular shape, but its geometry corresponds to a closed curveellipse. This form of double-lane roundabout is characterized by two semiaxes ( $R$ and $2 R$ ). Usually the higher semiaxis (2R) is located in the main traffic direction while the lower semiaxis is located in the secondary traffic direction. This form of elliptical roundabout is most often applied in areas where, due to spatial conditions, a classical circular roundabout cannot be performed and where there is more traffic at the main traffic direction. Mainly in the world it is not so much represented, but according to [8], elliptical roundabout is widely used in Iran. Figure 1 represents, respectively, the two geometries examined, considering the main and secondary traffic directions. Both road geometries are not signalized; therefore the precedence of vehicles circulating on the ring has been estimated with respect to those that are introduced by the arms in the various directions, respectively.

\section{Calibration of Microscopic Traffic Simulator: Methods and Application}

Microsimulation approach and microanalytic simulation are linked to a category of analytical tools that perform highly detailed analysis of activities such as highway traffic flowing through road networks. Traffic microsimulation models generally is defined considering a large number of parameters that must be calibrated before the prediction of applied model.

In this paragraph, the details of the traffic microsimulator used and the tool dedicated to the assessment of road safety have been reported. The definitions of the parameters and the calibration of the models followed the processing of the data and the comparison of the two geometries.

3.1. Microsimulation Tool Description. The modelling phase of the compared roundabouts was made by first considering the geometric parameters and the main and secondary traffic directions. It was carried out considering some hypotheses related to the models on which the VISSIM software developed by PTV is based.

The parameters mentioned above mainly include the characteristics of the vehicle, the composition of the traffic flow in terms of percentages of heavy or other light vehicles, the distribution of the speed of desire, the traffic flows, and some behavioural parameters linked to the style of driving and the driver's psychophysical status [9].

The simulated model considers a random generation in the distribution along the vehicle queues in terms of both composition and volume. It also considers that the priority of the fastest moving vehicle may decrease when it reaches and perceives the presence of a slower vehicle in front.

The basic concept of this model is that the driver of a faster moving vehicle starts to decrease own speed as he reaches his individual perception threshold to a slower moving vehicle. Vehicles would be randomly generated as per the given volume and composition.

In order to be able to evaluate surrogate security parameters using the SSAM specific tool, it is first necessary to generate a layout from VISSIM and through specific files in "trj" format possible to trace the trajectories of individual vehicles and therefore to the propensity that they have to enter in fact that the SSAM tool analyzes vehicle interactions to identify events and catalogue all the events found.

For each such event, SSAM also calculates several surrogate safety measures, including the following:

(i) Minimum time to collision (TTC)

(ii) Minimum postencroachment (PET).

The simulation of traffic through the VISSIM software initially considers the definition of traffic flows and their 

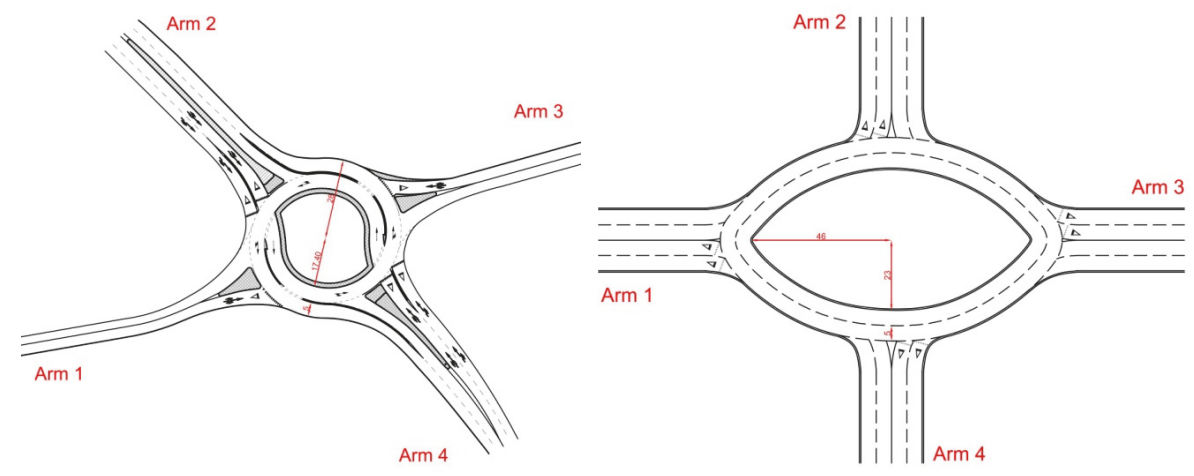

FIGURE 1: Details of turbo (left) and elliptical (right) geometry.

distribution along the intersection arms through the creation of special $\mathrm{O} / \mathrm{D}$ matrices. In the rotary intersections, the deflection curves created by the motion of the vehicles depend on the geometry of the roundabout itself and in particular on the size of the central island. Another important geometric parameter is the determination of the input radius of the branches on the circulatory ring. They depend on both the capacity and the safety of the examined scheme. The aforesaid parameter is related to the width of entry, the width of the carriageway on the circulatory ring, and the geometry of the central island. These parameters affect the travel speed of the vehicle and therefore the driving comfort and road safety.

Federal Highway Administration (FHWA) Roundabout Guidebook [10] was considered to select roundabout design parameters. After designing all of the roundabouts in VISSIM for both geometries and conditions, traffic loads were assigned. In this study, the actuated traffic control was used in roundabouts. In addition, the right-of-way control was considered for not signalized roundabouts that were given priority to circulating flow. This controlling system allowed roundabouts to perform better than uncontrolled systems. Each roundabout was designed considering double lane on circulatory carriageway.

The following assumptions were considered for this study are linked to urban areas conditions. The analyzed geometries are characterized by a speed value of about $50 \mathrm{~km} / \mathrm{h}$ on circulatory carriageway while the access speeds from each arm are assumed to be less than $35 \mathrm{~km} / \mathrm{h}$. In elliptical roundabouts, large radius is twice the small radius.

The exact disposition of the data and the traffic flows have been calibrated through the origins of origin and are examined in the next paragraphs.

In general, it is possible to resort to traffic microsimulation instruments if, in a preventive manner and for small areas, the possible introduction of different geometrical schemes is to be analyzed and can be compared from both the point of views of safety-related issues (i.e., precedence, percentage of thinking vehicles, and weak road users) in relation to impacts of various kinds such as the environment [11].

This research investigates on two unconventional geometrical layouts (elliptical and turbo roundabouts). They are very recent or just theoretic, and it is often not possible to perform an observational evaluation by collecting crash and operational data.

The microsimulation software tool VISSIM (Version 9) [1] was used to simulate operations at the roundabouts. This software can be used to generate all types of outputs simultaneously (safety, traffic performance, and capacity), allowing a more complete and comprehensive picture of the combined effects in making comparisons of various roundabout design scenarios.

The VISSIM output, in particular files linked to trajectories details, was imported into the Surrogate Safety Assessment Model (SSAM) [2], a software application designed to consider vehicle trajectory data output from microscopic traffic simulation models to derive proximal measures such as conflicts, based on thresholds for either time to collision (TTC) or postencroachment time (PET).

The estimated conflicts were then applied in crashconflict models for roundabouts developed in other research to compare the roundabout designs on the basis of expected crashes.

The approach of using proximal safety indicators such as conflicts has been suggested as an alternative to the use of crash data, especially where the facility being evaluated does not exist and thus does not have a crash record.

It is possible to consider the frequency of conflicts and the severity measures through the use of intersection conflict index in agreement with Sayed and Zein [12].

This suggests the possibility of using significantly shorter study periods to establish statistically reliable results. The fundamental parameters beyond those previously described are related to the capacity of the analyzed geometry, to the estimated or hypothesized traffic volume and to their relationship. The comparison of the results took place considering the geometries shown in the Table 1.

According to Hatami and Aghayan [8], it is possible to admit that the elliptic roundabouts have now become an unconventional type of roundabout.

The geometry of the aforesaid roundabout leads to a reduction of the positive effects especially with the increase of the radius: in fact, there is a roundabout an elliptic not semaphore, like that object of study, to an increase of the 
TABLE 1: Details of double-lane roundabouts simulated.

\begin{tabular}{|c|c|c|c|c|c|c|}
\hline Number of lanes & $\begin{array}{c}\text { Types of } \\
\text { roundabout }\end{array}$ & $\begin{array}{c}\text { Speed } \\
\text { limits }[\mathrm{km} / \mathrm{h}]\end{array}$ & Traffic light & $\begin{array}{l}\text { Central island } \\
\text { radii }[\mathrm{m}]\end{array}$ & $\begin{array}{c}\text { Total Traffic } \\
\text { Volume[veh/h] }\end{array}$ & $\begin{array}{c}\text { Max } \\
\text { Capacity }\end{array}$ \\
\hline Two lanes & Turbo & 50 & Absence & Main radius $\mathrm{R}=56 \mathrm{~m}$ & 3040veh/h & $3480 \mathrm{veh} / \mathrm{h}$ \\
\hline Two lanes & Elliptical & 50 & Absence & $\begin{array}{c}\text { Main radius } \mathrm{R}=56 \mathrm{~m} \\
\text { Secondary radius } \\
\mathrm{r}=23 \mathrm{~m}\end{array}$ & $2400 \mathrm{veh} / \mathrm{h}$ & $3000 \mathrm{veh} / \mathrm{h}$ \\
\hline
\end{tabular}

criticalities both in terms of capacity and of delay. Against the increase of the speed in it involves a positive effect both on the capacity and on the delay.

The Surrogate Safety Assessment Model (SSAM) is a specific tool created by Federal Highway Administration focused on identification, automatically classification of the presence of potential conflicts generated by the trajectories of vehicles circulating on road schemes. The tool also has integrated statistical analysis functions for the frequency and severity of conflicts that can help in the design of traffic safety structures. The following paragraphs describe the calibration steps of the model and report the relative results of the unidentified roundabout, providing the results with the help of the VISSIM software and the SSAM tool.

3.2. Surrogate Safety Tool SSAM. The name of SSAM tool is the acronym of Surrogate Safety Assessment Model. It has many parameters to configure that allow the user to visualize the analysis and customize the analysis results. Some of these data would be best imported from the simulation application(s) itself (e.g., "import traffic-stream data") using a common file format related to trajectories generated by microsimulator software.

Generally the data required to adequately analyze the traffic situation includes the following:

(i) Network geometry (i.e., number of lanes in each direction, turning pockets, and driveways)

(ii) Traffic-stream data (volume of traffic in each direction, change in the volumes over the simulation period)

(iii) Definition of signalized or not signalized intersection with specific priority of each arms or circulatory carriageway

(iv) Driver behaviour data (i.e., aggressiveness distribution and gap-acceptance criteria).

The evaluation of surrogate parameters allows an evaluation of the safety of the schemes analyzed, providing a greater propensity of the intersection to generate different types of conflict points.

3.3. Calibration Process to Microsimulation Tool. Calibration is the adjustment process to set model parameters and to improve the model's ability to reproduce local driver behaviour and traffic performance characteristics.

It is impossible for the researcher to have knowledge of the number of repetitions to be calibrated in advance, but it is possible to use an estimate of how many times it is necessary
TABLE 2: Minimum number of repetitions needed to obtain the desired confidence interval.

\begin{tabular}{lcc}
\hline $\begin{array}{l}\text { Desired Range } \\
(\mathrm{CI} / \mathrm{S})\end{array}$ & Desired Confidence & Minimum Repetitions \\
\hline 0,5 & $99 \%$ & 130 \\
0,5 & $95 \%$ & 83 \\
0,5 & $90 \%$ & 64 \\
1,0 & $99 \%$ & 36 \\
1,0 & $95 \%$ & 23 \\
1,0 & $90 \%$ & 18 \\
1,5 & $99 \%$ & 18 \\
1,5 & $95 \%$ & 12 \\
1,5 & $90 \%$ & 9 \\
2,0 & $99 \%$ & 12 \\
2,0 & $95 \%$ & 8 \\
2,0 & $90 \%$ & 6 \\
\hline
\end{tabular}

to calibrate the parameter and/or models investigated to obtain a statistically valid result. The required minimum number of model repetitions is computed using the following equation:

$$
\mathrm{CI}_{1-\alpha \%}=2 * \frac{\mathrm{t}_{(1-\alpha / 2), N-1} \mathrm{~s}}{\sqrt{ } N}
$$

where

$\mathrm{CI}(1-\alpha) \%=(1-\alpha) \%$ is confidence interval for the true mean, where alpha equals the probability of the true mean not lying within the confidence interval

$t(1-\alpha / 2), N-1$ is Student's t-statistic for the probability of a two-sided error summing to alpha with $\mathrm{N}-1$ degrees of freedom, where $\mathrm{N}$ equals the number of repetitions

$s$ is standard deviation of the model results.

Table 2 shows the link among above equation, the minimum number of repetitions for various desired confidence intervals, and desired degrees of confidence according to [2].

3.4. Calibration Targets. The model calibration process provides the best possible match between model performance estimates and real measurements.

However, there is a limit to the amount of time and effort that anyone can make to eliminate the error in the model, since the high repetition of the process often does not result in greater accuracy of the data.

Table 3 shows the criteria and calibration measures considering specific vehicle flow intervals adopted by DOT [13]. 
TABLE 3: Wisconsin DOT freeway model calibration criteria.

\begin{tabular}{lcc}
\hline Criteria and Measures & Traffic Flow range & Calibration Acceptance Targets \\
\hline & Individual Link Flows & $>85 \%$ of cases \\
Within 15\% & $700 \mathrm{veh} / \mathrm{h}<$ Flow $<2700 \mathrm{veh} / \mathrm{h}$ & $>85 \%$ of cases \\
Within 100veh/h & Flow $<700 \mathrm{veh} / \mathrm{h}$ & $>85 \%$ of cases \\
Within 400veh/h & Flow $>2700 \mathrm{veh} / \mathrm{h}$ & Within 5\%of sum of all link counts \\
Sum of All Link Flows & $>\mathbf{8 5} \%$ of cases \\
GEH statistic $<\mathbf{5}$ for Individual link Flow & GEH<4 for sum of all link counts \\
GEH statistic $<$ 5 for all link Flow & $>85 \%$ of cases \\
Travel times, Model Versus Observed & & To analyst's satisfaction \\
Journay Time, Network Within 15\%(or 1min if higher) & & To analyst's satisfaction \\
Visual Adults & & \\
Individual Link Speeds-visually Acceptable Speed-Flow relationship & \\
Bottlenecks & & \\
Visually Acceptable Queuing & &
\end{tabular}

TABLE 4: GEH value obtained in accordance with [4].

\begin{tabular}{|c|c|c|c|c|c|}
\hline \multicolumn{6}{|c|}{ Roundabout schemes with 2 lanes on circulatory carriageway } \\
\hline & Double lane & Turbo & Flower & Target & Elliptical \\
\hline GEH on right lane & 87,5 & 87,5 & 87,5 & 87,5 & 87,5 \\
\hline GEH on left lane & 100 & 87,5 & 87,5 & 87,5 & 87,5 \\
\hline
\end{tabular}

The calibration of the software model used is based on the model defined Wiedemann 74 used by PTV VISSIM (version 9) to simulate in a reliable and realistic way the traffic of vehicles inside unconventional roundabouts.

The car-following model was considered to be satisfactorily calibrated when the two curves overlap.

To further explore the model's validity, the Geoffrey E. Havers (GEH) statistic index $(1,2)$ was used as the criterion for accepting the template.

The formula for the "GEHi Statistic" is

$$
\mathrm{GEHi}=\frac{\sqrt{2(M-C)^{2}}}{M+C}
$$

And

$$
\mathrm{GEH}=\frac{\sum_{\mathrm{i}=1}^{\mathrm{n}} \mathrm{GEHi}}{\mathrm{n}}
$$

GEH index is a global indicator widely used for the validation of traffic simulation models, especially when only aggregate values such as traffic flow counts in time-based detection stations and input capacity are available [14].

The GEH index value is the average over " $n$ "; it was considered equal to 9 , simulations of the index for each simulation $\mathrm{i}$, as shown in Table 4.

3.5. Traffic Flow Conditions. Microsimulation approach is helpful to simulate different hypothesis of traffic operations changing schemes and different traffic conditions. The authors investigated the safety results of the two analyzed road schemes considering the $80 \%$ of the maximum value of traffic flow, corresponding to each roundabout capacity.
TABLE 5: O/D matrix selected to simulation.

\begin{tabular}{lcccc}
\hline O/D & Arm1 & Arm 2 & Arm 3 & Arm 4 \\
\hline Arm1 & 0 & 0.15 & 0.7 & 0.15 \\
Arm2 & 0.15 & 0 & 0.15 & 0.7 \\
Arm3 & 0.7 & 0.15 & 0 & 0.15 \\
Arm4 & 0.15 & 0.7 & 0.15 & 0 \\
\hline
\end{tabular}

The simulation of the geometries described above was carried out by evaluating a ratio between Volume and Capacity of each single intersection equal to 0.8 , thus equal to $80 \%$ of the maximum capacity of each scheme in a double lane examined.

In general, the performances of the road geometry are measured through its level of service while the safety measures can consider surrogate parameters such us the time to collision (TTC). This parameter is obtained considering the trajectories of the individual vehicles and the potential conflicts. Through the TTC it is possible to provide interesting information in advance, suggesting that the new layouts should be considered where justified by considerations on costs and benefits. The VISSIM output includes vehicle trajectory files that were then imported into the Surrogate Safety Assessment Model-SSAM, a software application designed to perform analysis of vehicle trajectory data output from microscopic traffic simulation models to derive proximal measures such as conflicts, based on time to collision (TTC) or postencroachment time (PET). The estimated conflicts were then applied considering conflict models already analyzed by other sources of literature. The approach of using proximal safety indicators such as conflicts has been suggested as an alternative to the use of crash data, especially where the facility being evaluated does not exist and thus does not have a crash record. In particular the flow values used are equal to $80 \%$ of the saturation flux of each of the two rotary geometries. In particular, reference was made to a symmetric O/D matrix for both roundabouts and shown as on Table 5 .

The analyzed traffic mix refers to observed traffic distributions and consists of the following percentages: 70\% of LPV 

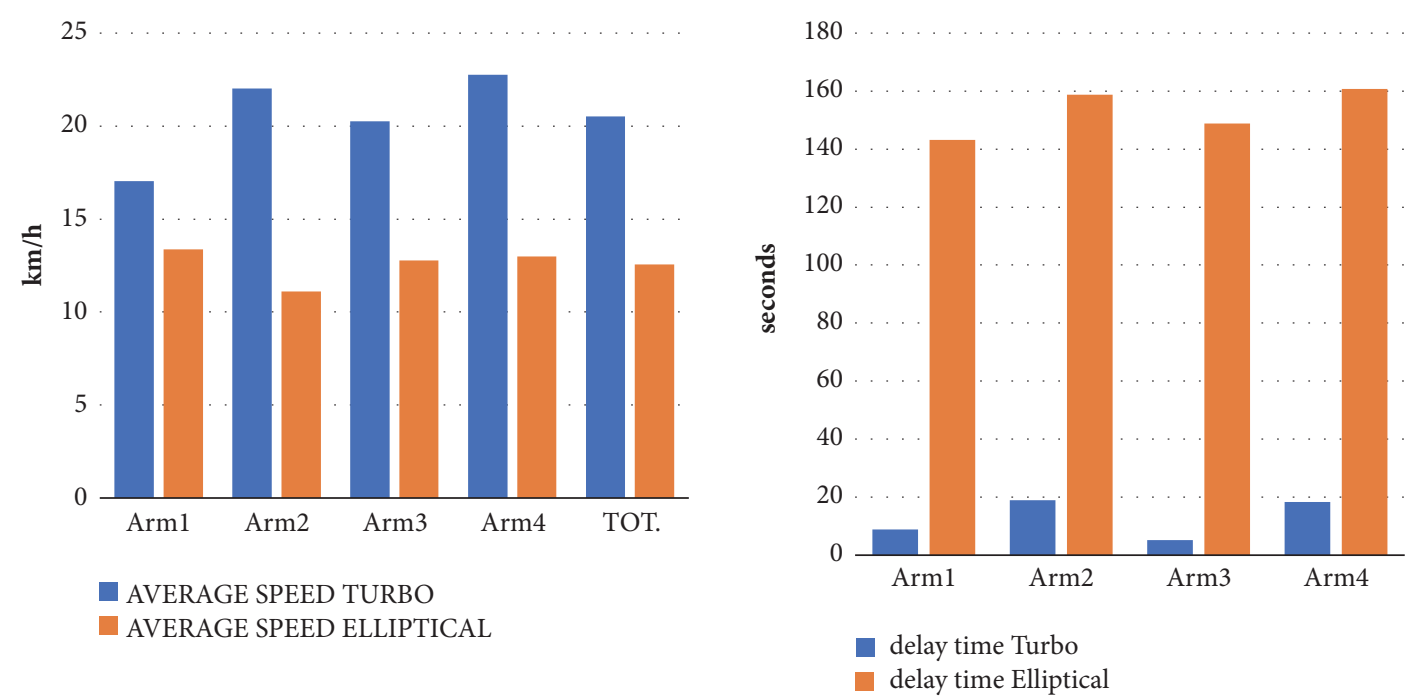

FIgURE 2: Comparison of average speed and delay time for investigated road scheme on saturated conditions.

(Light Passenger Vehicle), 20\%of HPV (Heavy Passenger Vehicle, $5 \%$ of Buses and the same percentage is dedicated to Motorcycles. The comparison of the geometries was carried out by evaluating the parameters characterizing the LOS service level of the road intersections and in particular it was estimated: maximum and average length of the queues, average delay, average speed, and number of STOPs. In order to make the two geometries comparable, reference is made to normalized values.

\section{Elliptical and Turbo Roundabout Operational Safety Results}

Two schemes with two-lane circulatory ring were presented in this work. The choice of one or the other scheme depends on several factors. In general, an elliptical roundabout is a good choice when constraints such as precedence, existing road alignments, buildings, and/or wetlands affect the shape. As regards the turbo roundabout scheme, the separation of directional flows must be underlined through specific vertical signs or curbs [15].

In general, the physical separation of traffic lanes is interrupted only at entry points in the internal circulating carriageway. Since the weaving on the roundabout is no longer possible, drivers should be assisted by clear signage and lane signage.

4.1. Comparison of VISSIM Results. The simulation carried out with the VISSIM software, made it possible to compare the intersections described above through the evaluation of parameters relating to the service level of an infrastructure. In particular, the length of the queues, delays and the stops number were evaluated. These parameters make it possible to evaluate how much a road geometric scheme has, for a given vehicular flow, the propensity to be subject to congestion phenomena and therefore to a low level of service. This status implies the possible collapse of the mobility of the vehicles inside it. Graphs on Figure 2 shows a sharp difference in the delay values along the two geometries with a very high possibility of congestion in the case of the elliptic roundabout compared to the turbo type.

The graph therefore shows a higher average speed along the turbo-rotary because the preselection of the manoeuvres does not compromise the travel speed of the entire roundabout. It shows a total average speed value of about 20 $23 \mathrm{~km} / \mathrm{h}$ for the turbo-type roundabout and about a reduced value of $40 \%$ for the elliptical roundabout. Likewise, it assumes almost zero or reduced delay values in the turbo-type intersection, while elevating values for the elliptical.

As far as the number of stops is concerned, there is a greater value in the arms B and D of the turbo roundabout, while they are practically of equal value along the elliptical roundabout in accordance with Figure 3. This phenomenon provides an increase in congestion and possible impacts in the aforementioned points.

An operational analysis is conducted to determine if the turbo roundabout will accommodate projected traffic volumes at an acceptable level of service (LOS). Roundabout LOS is measured in control delay consistent with the elliptical roundabout.

It is also fundamental to be able to understand the service level of the analyzed intersections, to evaluate the LOS parameter overall and/or for each arm. As can be seen from the graph shown in Figure 4, the value of LOS afferent to the elliptical roundabout remains almost constant along the arms for the traffic conditions previously discussed. In particular, the $\mathrm{LOS}=\mathrm{F}=6$ shows a bad condition of internal movement of this scheme in the roundabout, suggesting a widespread congestion of vehicular traffic to the flow analyzed. On the contrary, as regards the turbo-type roundabout, there is a more acceptable value of LOS, especially in arms 1 and 3 where the accumulated delays and the phenomenon of congestion seem to decrease by about $40 \%$ compared to two arms, 2 and 4 . 


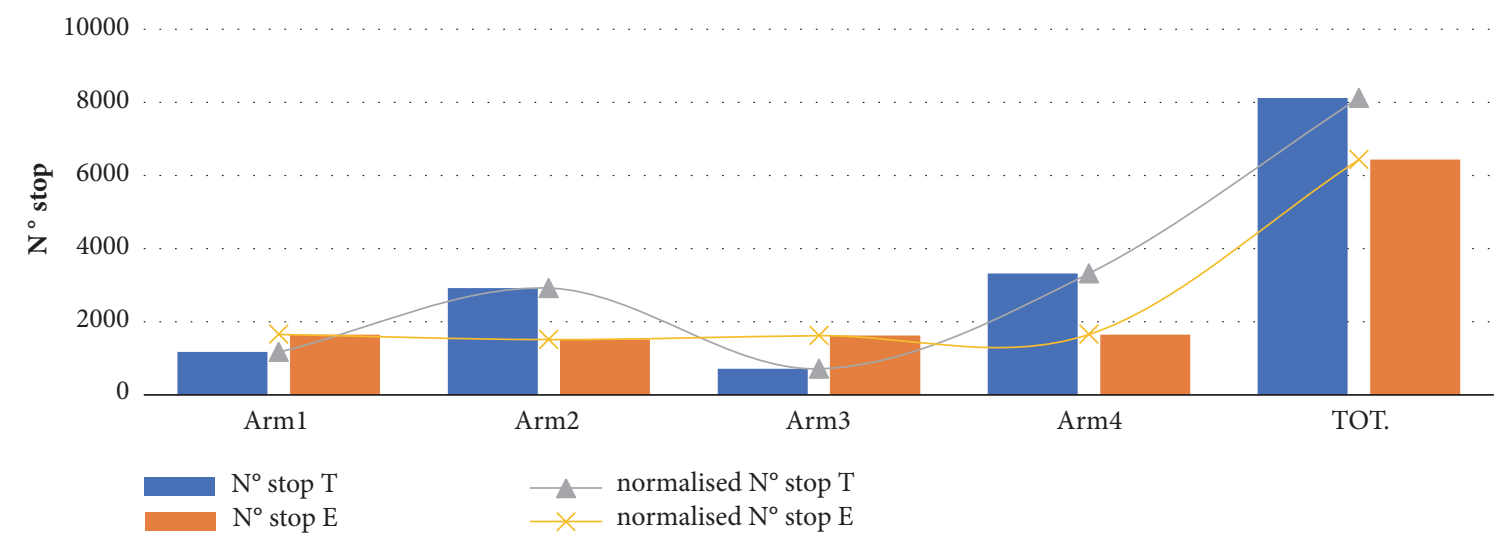

FIGURE 3: Comparison of number of stop related to turbo and elliptical scheme on congested conditions.

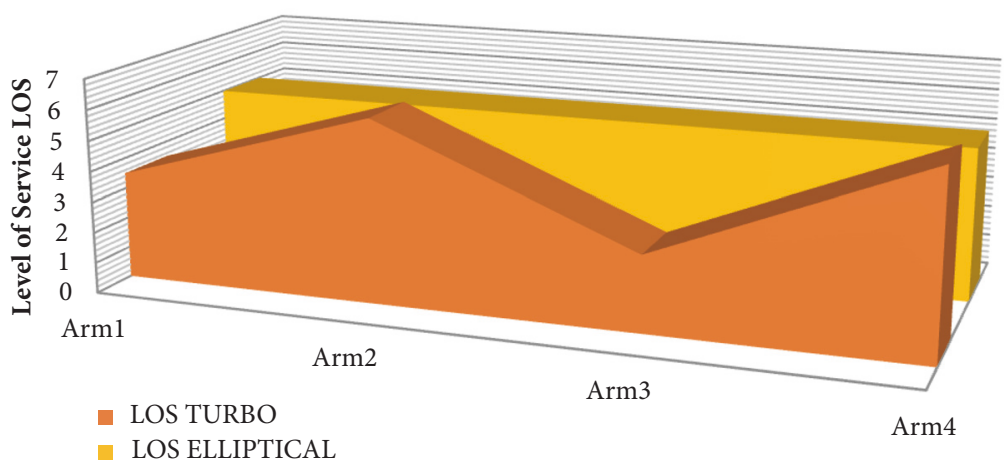

FIGURE 4: Level of service of investigated road schemes at grade.

The greater extension of the diameter along the main direction Arm1_Arm3 of the elliptical roundabout shows through the layouts produced by the microsimulator on Figure 5 a free flow condition near the circulatory carriageway while there is a greater thickening of vehicles along the arms for both the examined configurations. The lack of separation of the lanes on the main ring generates more collisions in the elliptical roundabout as explained in Figure 5.

4.2. Comparison of SSAM Results. TTC and PET are both indicators of proneness to establish a vehicle collision.

The probability of a conflict occurring is greater than the value of these parameters is lower [15]. The research was founded on specific range for both safety parameters in order to establish the possibility of defining a potential conflict, considering $0.1 \mathrm{~s}<\mathrm{TTC}<1.5 \mathrm{~s}$ and $0.5 \mathrm{~s}<\mathrm{PET}<2.5 \mathrm{~s}$. Among the data associated with each conflict is a MinTTC variable, which is the simulation time where the minimum TTC value for that conflict was observed [16]. That variable was used to filter out all conflicts that occurred during the simulation time. Two comparative safety evaluations were conducted based on different measures like described on Figure 6:

(i) The estimated conflicts from SSAM: this is done for entry flows of $0.8 \mathrm{~V} / \mathrm{C}$ (absolute and normalized per 1000 vehicles) and for $2400 \mathrm{veh} / \mathrm{h}$ and $3040 \mathrm{veh} / \mathrm{h}$. (ii) Estimated crashes are based on applying estimated conflicts (for entry flows of $0.8 \mathrm{~V} / \mathrm{C}$ and for 3040 $\mathrm{veh} / \mathrm{h}$ ) in crash-conflict models; as can be seen from Figure 6, it assists a maximum PET value for both the turbo and elliptic type scheme below the value of 5 . The average value, on the contrary, of results for the elliptic configuration is about $30 \%$ of the value average relative to the turbo. In terms of PET, there is a clear variability between the two geometries in terms of both maximum value and average value. In particular, the maximum value of the elliptical roundabout reaches a value equal to 5 determining an increase in the possible conflicts within it. Figure 6 shows the distribution of TTC and PET values considering turbo and elliptical scheme. In terms of average values it is possible to consider the high value of TTC for turbo and the same history for PET value.

We can notice a greater distribution in the arms, especially in the main direction with regard to the turbo-type roundabout, while an increase in yellow at the rotary crown couplings as regards the elliptical roundabout, equally distributed along the two directions. Equal distribution in the elliptic is denoted with regard to the conflicts shown in blue which instead are lower in the roundabout of the turbo type and bordering only in part of the lanes dedicated to the manoeuvre of turning to the right. Table 6 shows the comparison of the points 

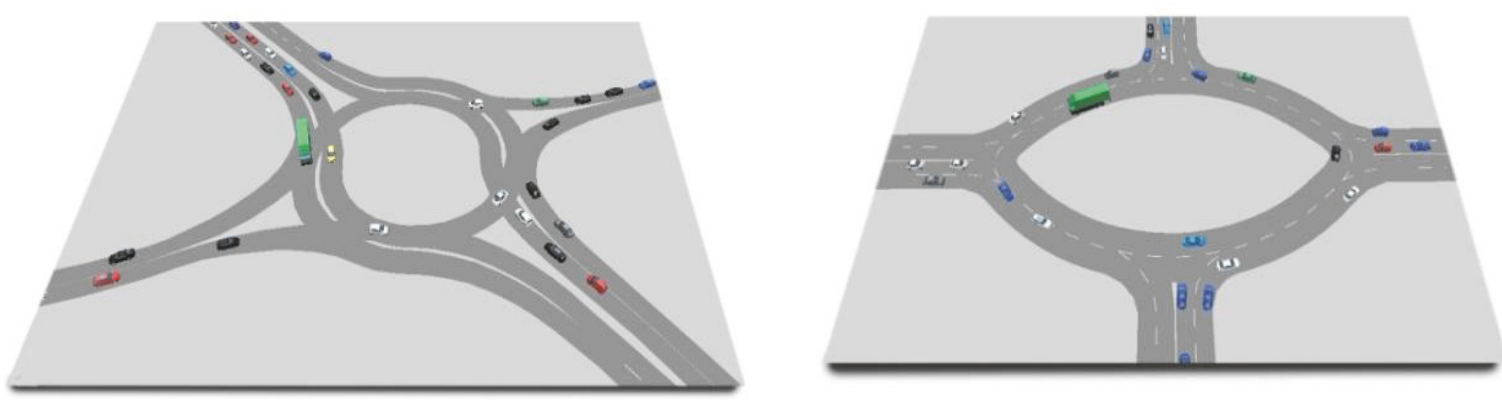

FIGURE 5: Microsimulator layout.
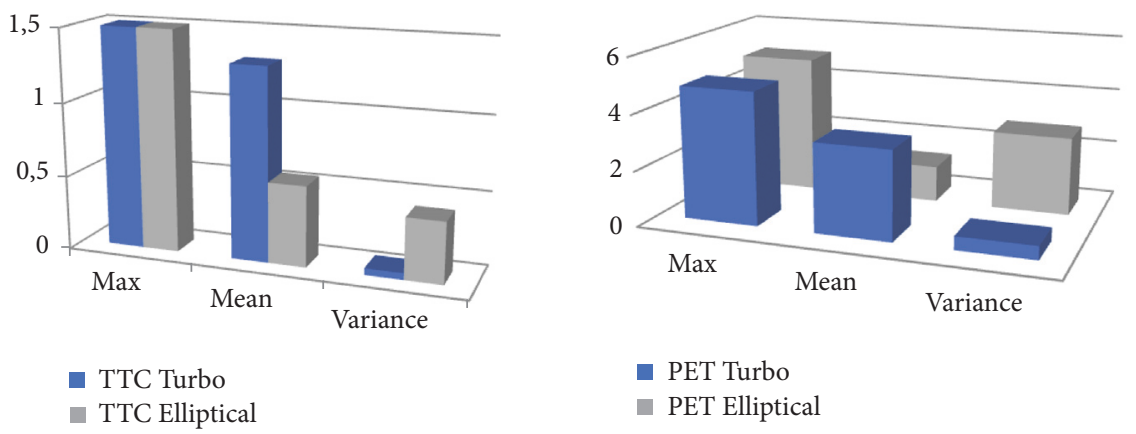

FIGURE 6: Surrogate safety parameters comparison: TTC and PET.

TABLE 6: Collision type distribution.

\begin{tabular}{lccc}
\hline Intersection scheme & \multicolumn{3}{c}{ Collision Type } \\
\hline $\begin{array}{l}\text { Turbo } \\
\text { Total conflict point 12173 }\end{array}$ & 7 & rear end & lane change \\
$\begin{array}{l}\text { Elliptical } \\
\text { Total conflict point 11528 }\end{array}$ & 453 & 8002 & 145 \\
\hline
\end{tabular}

of conflict for the various manoeuvres on the two types of intersections investigated.

Through the graphic representation of the values mentioned in Table 6 along the geometry, it is better to see Figure 7 in the arms of direct turning of the turbo the types of conflict called rear end while in the elliptical roundabout the same types of conflict are thickened inside the rotary crown near the access arms.

In the elliptical roundabout, also in Figure 7, there is a concentration of the conflicts of the wool change type at the coupling between the circulatory carriageway and the entry arms.

\section{Conclusions}

This research investigated two alternatives type of doublelane roundabout, turbo and elliptical, in order to define the safest solution considering direct and surrogate parameters.
To date, mutual comparisons in terms of surrogate safety between these two schemes are not discussed in the sector literature but can be compared to other roundabout geometries and or in terms of capacity-delay/geometrical parameters.

The work highlights the potential for estimating road safety through the replacement solution. Therefore the research work carried out wanted to highlight the negativity of one or the other scheme in order not only to compare two but to provide more comprehensive data that can be incorporated into the comparison of more geometries and also to help to technicians or local authorities during the preliminary selection phase of infrastructure projects.

The assessment of the critical aspects related to road safety and to the possible collision of vehicles has been addressed through the use of traffic microsimulation and a dedicated tool.

The hypotheses analyzed consider the presence of a double lane on the ring that if it does not have the curb of separation between them, it allows a greater percentage of collisions due to the lane change manoeuvres along the central ring. In fact the preselection of the manoeuvring lane along the turbo entry arm limits the collision formations for lane change. The evaluation of safety surrogate parameters such as TTC and PET allows analyzing in a preliminary phase the criticalities found in particular geometries and providing the bases to local authorities in order to choose, with the same geometry as a satin, which can generate the maximum reliable value of collisions even in critical conditions of saturation.

Finally, it should be noted that the presence of more points of collision within the circulatory ring can make traffic 

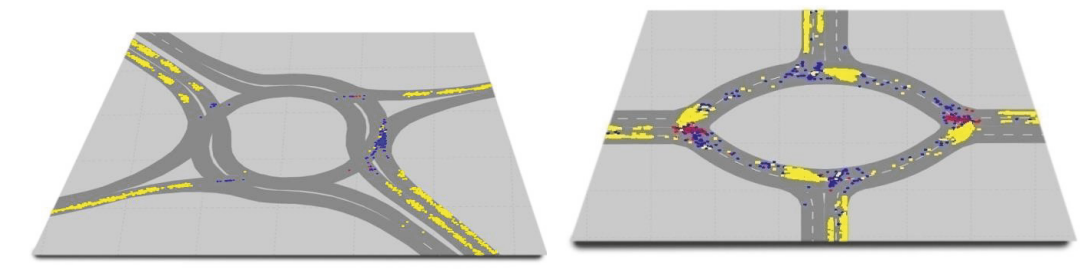

\begin{tabular}{|c|c|}
\hline \multicolumn{2}{|c|}{ Legend } \\
\hline Graphical representation & Conflict type \\
\hline Blue dots & Lane-change \\
Yellow dots & Rear-end \\
Red dots & Crossing \\
\hline
\end{tabular}

FIGURE 7: Layout related to the types of collisions made by SSAM in the two roundabouts examined.

less easy, especially if more lanes are invaded. These reasons are to evaluate in advance preventive scenarios and possible alternatives and solutions to the problems in progress.

\section{Limitations and Future Development}

This research is focused on the first phase of comparison in terms of safety of turbo and elliptical roundabout geometries in critical conditions of vehicular outflow with symmetrical distribution along the main and secondary directions. Future developments will be conducted varying not only transport matrices but also percentages of heavy vehicles.

\section{Data Availability}

The authors declare themselves available to provide input and output data of the present research work.

\section{Disclosure}

This research work is not a public or private expense for its elaboration. No research project of the Kore University of Enna, Italy, and the University of Maribor, Slovenia, has financed this work.

\section{Conflicts of Interest}

The authors declare that there are no conflicts of interest regarding the publication of this paper.

\section{References}

[1] J. Archer and I. Kosonen, "The Potential of Micro-Simulation Modelling in Relation to Traffic Safety Assessment," in Proceedings of the ESS Conference, Hamburg, Germany, 2000.

[2] T. Campisi, G. Tesoriere, and A. Canale, "Microsimulation approach for BRT system: The case study of urban turbo roundabout," in Proceedings of the International Conference of Computational Methods in Sciences and Engineering 2017, ICCMSE 2017, Greece, April 2017.

[3] H. Chin and S. Quek, "Measurement of Traffic Conflicts," Safety Science, vol. 26, pp. 169-185, 1997.
[4] R. Souleyrette and J. Hochstein, Development of a Conflict Analysis Methodology Using SSAM; Final Report, Center for Transportation Research and Education, Iowa State University, Ames, IA, USA, 2012.

[5] O. Feldman, "The GEH measure and quality of the highway assignment models," in Proceedings of the European Transport Conference, Glasgow, UK, 2012.

[6] L. G. H. Fortuijn, “Turbo roundabouts: Design principles and safety performance," Transportation Research Record: Journal of the Transportation Research Board, no. 2096, pp. 16-24, 2009.

[7] D. Gettman, L. Pu, T. Sayed, and S. Shelby, "Surrogate safety assessment model and validation," FHWA-HRT-08-051, 2008.

[8] T. Giuffrè, S. Trubia, A. Canale, and B. Persaud, "Using microsimulation to evaluate safety and operational implications of newer roundabout layouts for European Road networks," Sustainability, vol. 9, no. 11, 2017.

[9] R. Guide, "Wisconsin Department of Transportation," 2006.

[10] H. Hatami and I. Aghayan, "Traffic efficiency evaluation of elliptical roundabout compared with modern and turbo roundabouts considering traffic signal control," Promet - Traffic \& Transportation, vol. 29, no. 1, pp. 1-11, 2017.

[11] F. Huang, P. Liu, H. Yu, and W. Wang, "Identifying if VISSIM simulation model and SSAM provide reasonable estimates for field measured traffic conflicts at signalized intersections," Accident Analysis \& Prevention, vol. 50, pp. 1014-1024, 2013.

[12] PTV-Vision, VISSIM 5.30-05 user manual, 2011.

[13] L. Rodegerdts, "Roundabouts: An informational guide," Transportation Research Board, vol. 672, 2010.

[14] T. Sayed and S. Zein, "Traffic conflict standards for intersections," Transportation Planning and Technology, vol. 22, pp. 309$323,1999$.

[15] T. Tollazzi, R. Mauro, M. Guerrieri, and M. Renčelj, "Comparative analysis of four new alternative types of roundabouts: "Turbo", "flower", "target" and "four-flyover" roundabout," Periodica Polytechnica Civil Engineering, vol. 60, no. 1, pp. 5160, 2016.

[16] T. Tollazzi, G. Tesoriere, M. Guerrieri, and T. Campisi, "Environmental, functional and economic criteria for comparing "target roundabouts" with one- or two-level roundabout intersections," Transportation Research Part D: Transport and Environment, vol. 34, pp. 330-344, 2015. 


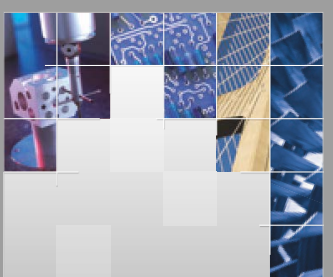

\section{Enfincering}
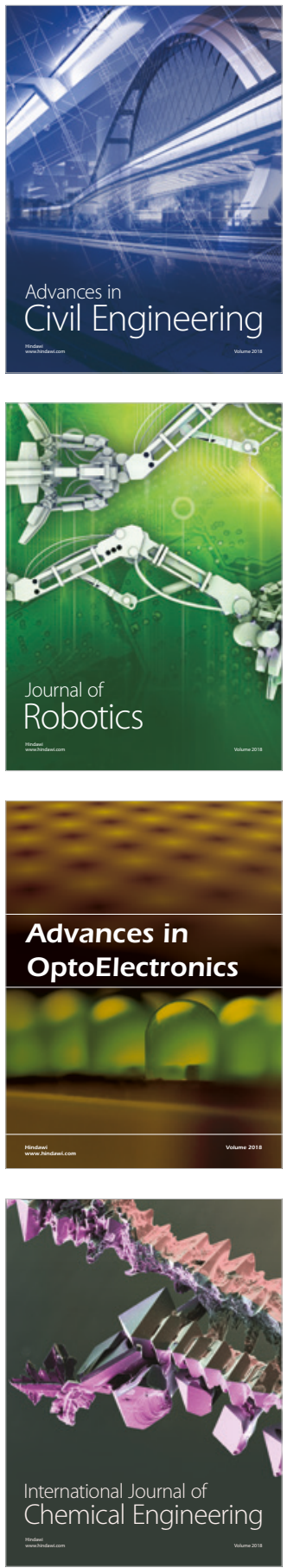

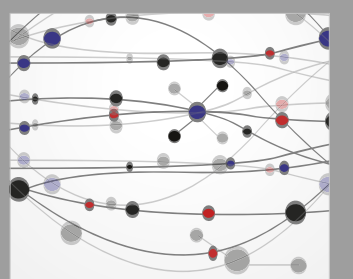

\section{Rotating \\ Machinery}

The Scientific World Journal

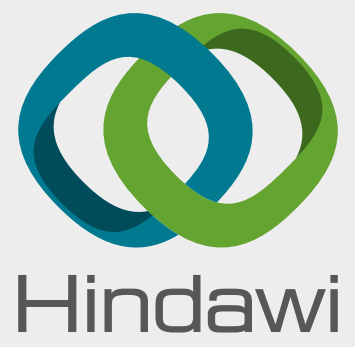

Submit your manuscripts at

www.hindawi.com
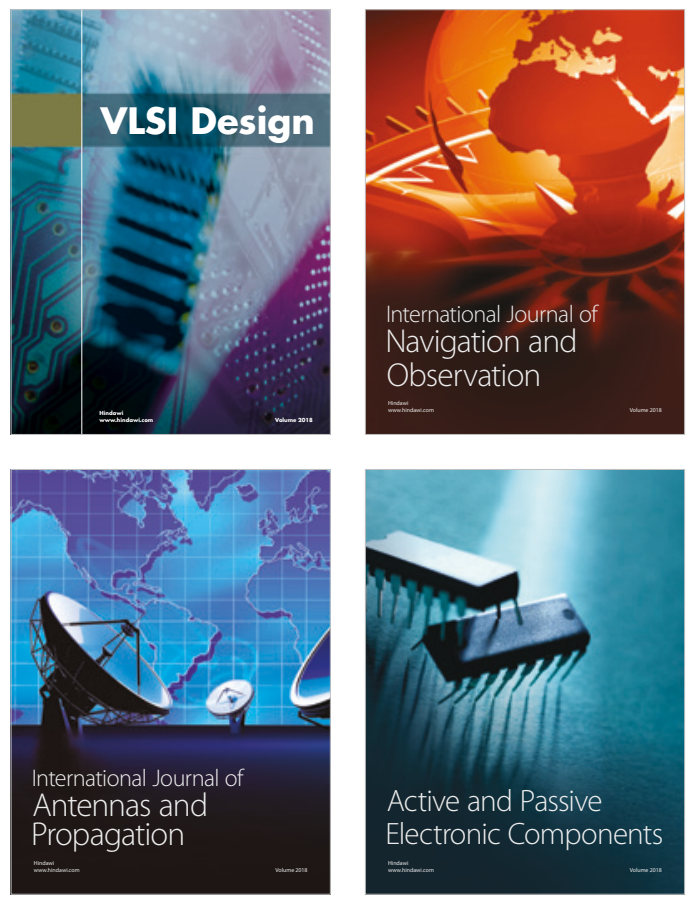
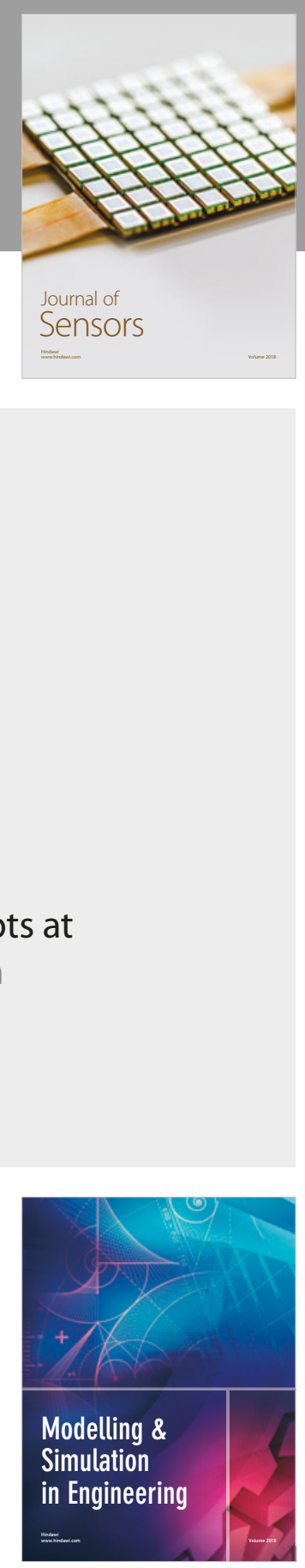

\section{Advances \\ Multimedia}
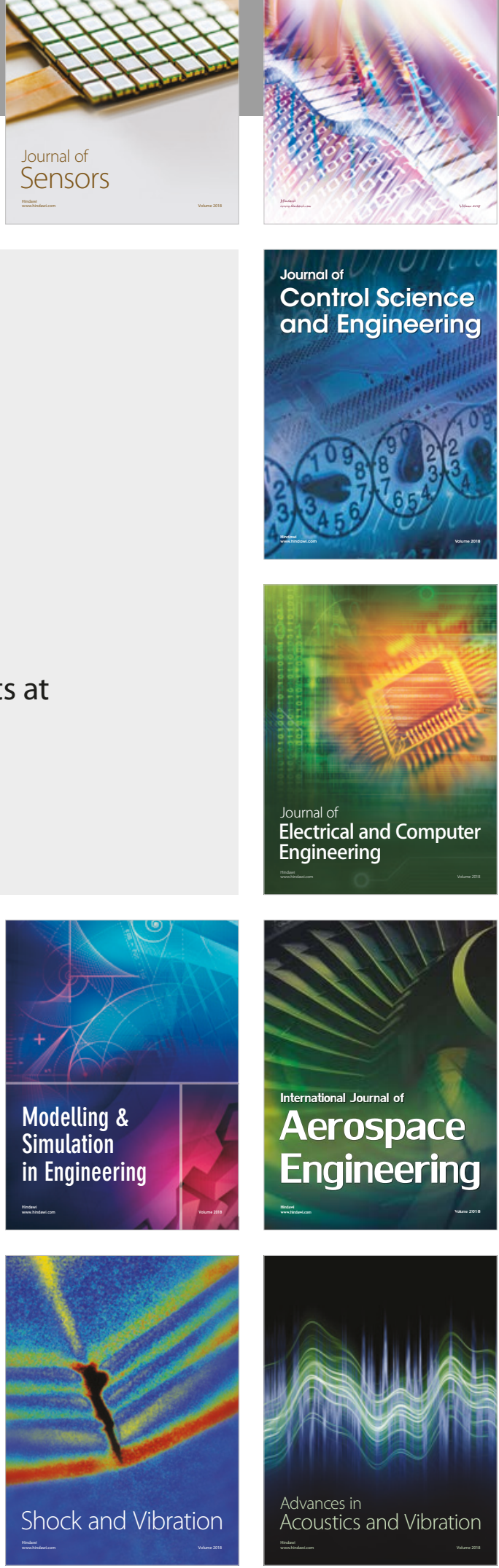